\title{
Depth perception in patients with congenital color vision deficiency
}

\author{
Serdar Ozates $\mathbb{1}^{1} \cdot$ Mehmet Ali Sekeroglu $^{2} \cdot$ Cagri Ilhan ${ }^{3} \cdot$ Sibel Doguizi $^{2} \cdot$ Pelin Yilmazbas $^{2}$
}

Received: 9 November 2017 / Revised: 4 July 2018 / Accepted: 1 October 2018 / Published online: 5 December 2018

(c) The Royal College of Ophthalmologists 2018

\begin{abstract}
Purpose To assess the effect of type and severity of congenital color vision deficiency (CCVD) on depth perception. Methods Thirty-one male patients with a known diagnosis of CCVD were included in the study group and 31 age-matched healthy subjects in the control group. After standard ophthalmological examination including best corrected visual acuity (BCVA) testing with Snellen chart, slit-lamp examination, non-contact tonometry, and fundus examination, all patients underwent color perception testing with Hardy-Rand-Rittler (HRR) 4th edition pseudoisochromatic test plates and stereoacuity testing with Titmus stereo test plates.

Results Of the 31 patients with CCVD, 7 were protanope and 24 were deuteranope. Mean stereoacuity was $46.77 \pm 11.3$, 105.7 \pm 69.0 , and $134.1 \pm 115.2$ in the control, protanope, and deuteranope groups, respectively. Stereoacuity was significantly better in the control group than in the protanope and deuteranope groups $(p=0.039, p<0.001$ respectively). No significant difference was observed between protanopes and deuteranopes regarding stereoacuity $(p=0.73)$. Mean BCVA was $-0.01 \pm 0.03,-0.02 \pm 0.07$, and $-0.10 \pm 0.11$ in the control, protanope, and deuteranope groups, respectively. Mean BCVA in deuteranopes was significantly better than the control group $(p=0.004)$, while mean BCVA in deuteranopes and protanopes did not differ significantly $(p=0.056)$. No significant difference was observed between the control group and protanopes regarding visual acuity $(p=0.921)$.

Conclusions Our study showed that color vision had an important effect on depth perception and CCVD may cause decreased stereoacuity.
\end{abstract}

\section{Introduction}

Stereopsis is an impression of depth arising from neural and cortical activities triggered by disparity between the right and left eyes [1,2]. The relation between color vision and stereopsis, and its physiology have been a point of interest for a long time. Luminance and color perception were suggested to be the main cues for depth perception, and several studies emphasized the importance of color perception on stereoacuity [3, 4]. Con-

Serdar Ozates

serdarozates@gmail.com

1 Department of Ophthalmology, Sami Ulus Maternity and Children Training and Research Hospital, Ankara, Turkey

2 Department of Ophthalmology, Ulucanlar Eye Training and Research Hospital, Ankara, Turkey

3 Department of Ophthalmology, Hatay State Hospital, Hatay, Turkey genital color vision deficiency (CCVD) is an inherited disorder, which causes a disturbance in the discrimination of different colors [5]. The discrimination of color depends on the unequal stimulation of three cone types and comparison of their signal properties [5, 6]. However, the physiological process stages of cone signals remain a controversial subject. In the literature, the association between color vision and depth perception has been investigated in numerous experimental and physiological studies, which were conducted on healthy persons. However, diseases may also provide unique information about how a normal physiological process works. Due to abnormal cone activity in CCVD, comparing their activity with normal cones may provide information about how abnormal or absent cone signal affects stereopsis. Therefore, this comparison may provide a different point of view on how the color discrimination process affects depth perception.

In this study, we aimed to assess how CCVD and its grade of severity affects depth perception and to reconsider the physiological mechanisms in the light of our findings. 
Table 1 Mean visual acuity and stereoacuity outcomes, and comparison of the groups

\begin{tabular}{|c|c|c|c|c|c|}
\hline & \multirow{2}{*}{$\begin{array}{l}\text { Protanopes }(n=7) \\
\text { Severe }\end{array}$} & \multicolumn{2}{|l|}{ Deuteranopes $(n=24)$} & \multirow[t]{2}{*}{ Control $(n=31)$} & \multirow[t]{2}{*}{$P$-value } \\
\hline & & Moderate $(n=8)$ & Severe $(n=16)$ & & \\
\hline $\begin{array}{l}\text { Mean visual acuity } \\
\text { (logMAR) }\end{array}$ & $\begin{array}{l}-0.02 \pm 0.07(\min : 0 \\
\max :-0.20)\end{array}$ & $\begin{array}{l}-0.08 \pm 0.12 \text { (min: } 0, \max : \\
-0.30)\end{array}$ & $\begin{array}{l}-0.11 \pm 0.11(\min : 0 \\
\max :-0.30)\end{array}$ & $\begin{array}{l}-0.01 \pm 0.03(\min : 0 \\
\max :-0.10)\end{array}$ & $0.005^{*}$ \\
\hline $\begin{array}{l}\text { Mean stereo acuity (arc } \\
\text { sec) }\end{array}$ & $\begin{array}{l}105.7 \pm 69.0(\min : 40 \\
\max : 200)\end{array}$ & $\begin{array}{l}113.7 \pm 120.5 \text { (min: } 40 \\
\max : 400)\end{array}$ & $\begin{array}{l}144.7 \pm 115.5(\min : \\
40, \max : 400)\end{array}$ & $\begin{array}{l}46.7 \pm 11.3 \text { (min: } 40 \\
\max : 80)\end{array}$ & $<0.001^{*}$ \\
\hline \multirow[t]{12}{*}{ Post hoc comparisons } & \multirow[t]{6}{*}{ Visual acuity } & Protanopes-deuteranopes & 0.056 & & \\
\hline & & Protanopes-control & 0.921 & & \\
\hline & & Deuteranopes - control & $0.004^{*}$ & & \\
\hline & & $\begin{array}{l}\text { Moderate deuteranopes- } \\
\text { control }\end{array}$ & 0.120 & & \\
\hline & & Severe deuteranopes - control & $0.002 *$ & & \\
\hline & & $\begin{array}{l}\text { Severe deuteranopes- } \\
\text { moderate deuteranopes }\end{array}$ & 0.411 & & \\
\hline & \multirow[t]{6}{*}{ Stereoacuity } & Protanopes-deuteranopes & 0.073 & & \\
\hline & & Protanopes-control & $0.039 *$ & & \\
\hline & & Deuteranopes-control & $<0.001 *$ & & \\
\hline & & $\begin{array}{l}\text { Moderate deuteranopes- } \\
\text { control }\end{array}$ & $0.016^{*}$ & & \\
\hline & & Severe deuteranopes — control & $<0.001 *$ & & \\
\hline & & $\begin{array}{l}\text { Severe deuteranopes- } \\
\text { moderate deuteranopes }\end{array}$ & 0.406 & & \\
\hline
\end{tabular}

$\log M A R$ logarithm of minimum angle of resolution, arc sec seconds of arc

*Statistically significant

\section{Material and methods}

This observational prospective study was conducted at Ulucanlar Eye Training and Research Hospital, Ankara, Turkey, in accordance with the ethical standards of the Declaration of Helsinki. The study protocol was approved by the institutional board of the Numune Training and Research Hospital ethics committee, Ankara, Turkey. All participants granted us an informed written consent prior to clinical investigations.

The patients who were admitted to our clinic with a previous diagnosis of CCVD between April 2015 and January 2017 were included in the study. Thirty-one male patients with CCVD were included in the study group and 31 healthy male subjects were included in the control group. Standard ophthalmological examination including best corrected visual acuity (BCVA) testing with Snellen chart, non-contact tonometry, slit-lamp examination, and fundus examination were performed for both study and control subjects. BCVA was converted to logarithm of minimum angle of resolution (logMAR) for statistical analysis. Complete strabismus examination was also performed including cover-uncover test, alternate cover test, Worth four-dot test, and the four-diopter base-out prism test. Exclusion criteria were history of any ocular surgery, trauma, disease including cataract, glaucoma, uveitis, retinal disorders, strabismus, microtropia, amblyopia, nystagmus, any type of refractive error greater than six diopters, and a history of optic nerve and central nervous system disorders.

Color perception and stereoacuity were tested by the same person (CI) using the Hardy-Rand-Rittler (HRR) 4th edition pseudoisochromatic test plates and Titmus stereo test plates (Stereo Optic Co. Inc., IL, USA), respectively. All tests were performed in the same room, which was illuminated by standard daylight fluorescent tubes $(5500 \mathrm{~K})$. The HRR test plates were placed $600 \mathrm{~mm}$ away and Titmus test plates were placed $400 \mathrm{~mm}$ away from the face plane of the patients, respectively.

Patients were allowed 3 seconds to respond to each plate and their responses were noted. No change of any patient's response was allowed. Six correct response to screening plates of HRR test revealed normal color vision. Errors on screening plates revealed CCVD and subsequent tests were performed to reveal the type and severity of CCVD as previously explained in detail [7]. Graded circles in the Titmus stereo test were used to determine fine depth perception as previously explained in detail [8]. Titmus test outcome noted as seconds of arc (arc sec).

Statistical analyses were performed with SPSS Statistics (Version 22.0, Armonk, NY: IBM Corp.). The assumption 
of normal distribution of data was tested by the ShapiroWilk $W$ test. Descriptive statistics of visual acuity and stereoacuity parameters were performed. Differences between the study groups were tested with the Mann-Whitney $U$ test and the Kruskal-Wallis test for independent samples. After multiple comparisons, Bonferroni correction was used.

\section{Results}

Thirty-one male patients with CCVD were included in the study group and 31 healthy male subjects were included in the control group. Of the patients with CCVD, 7 were protanopes and 24 were deuteranopes. The mean age was $26.1 \pm 11.1$ years (min: 11, max: 45 ) in protanopes, $26.4 \pm$ 9.8 years (min: 8 , max: 46) in deuteranopes, and $25.7 \pm 10.1$ years (min: 9, max: 48) in the control group. No significant difference was found between groups regarding age $(p=$ 0.953). Patients in study groups were also divided into subgroups according to severity of CCVD. All 7 patients in the protanope group had severe CCVD. In the deuteranope group, 8 patients had moderate and 16 patients had severe CCVD.

Table 1 shows the mean visual acuity and stereoacuity outcomes, and comparison of the groups. Comparison between the control group and study groups revealed that mean visual acuity in deuteranopes was significantly better than the control group. Mean visual acuity showed no significant difference between protanopes and deuteranopes. Comparison between severity subgroups of deuteranopes and the control group showed that visual acuity was significantly better in severe deuteranopes. Mean visual acuity did not differ between severe and moderate deuteranopes. Mean stereoacuity in the control group was significantly better than in the study groups. Also, significant difference was observed between severity subgroups of deuteranopes and the control group, in which stereopsis was better. No significant difference was observed between protanopes and deuteranopes, and between severity subgroups of deuteranopes regarding stereoacuity.

\section{Discussion}

The relation between color vision and stereopsis has long been a controversial subject, however, recent studies have proved cone inputs are a component of the depth perception process like luminance [9-11]. Our study revealed that mean stereoacuity in the control group was better than in the CCVD subgroups supporting the suggestion of Jordan et al. [10], which emphasized that cone inputs were influencing depth perception at some point of the visual pathway. Depth perception requires matching of two retinal images and significant cortical processing [12, 13]. Previous studies revealed the existence of neurons that are capable of utilizing both color and disparity [2, 14, 15]. Although the Titmus test uses polarized light to generate a sense of depth, polarized light contains all wavelengths and the optic media of the eye cause chromatic aberration. Chromatic aberration causes chromatic defocus and a retinal image consisting of several layers, which is characterized by different light wavelengths [16-18]. Chromatic defocus of the retinal image is an important cue for color disparity $[19,20]$. The responses of $\mathrm{L}$ and $\mathrm{M}$ cones to chromatic defocus vary due to the spectral sensitivity of these cones, and this difference encodes a different retinal image with different spatial locations resulting in color disparity [16]. Previous studies have shown the beneficial effect of the color disparity cue on depth perception [17, 18, 21]. However, patients with CCVD may fail to detect color disparity due to the absence of one of the cone pigments, resulting in decreased stereoacuity. Our study showed that defective color perception influenced stereoacuity in patients with CCVD. Although many experimental and physiological studies have reported that color vision has an impact on depth perception, our study is the first to show the stereoacuity change in the absence of L-cone or M-cone photoreceptor stimulus.

In our study, we found a wide range of stereoacuity outcomes in the CCVD patients in comparison to normal subjects. While retinal disparity is the main cue for depth perception, several other cues, such as accommodation, vergence eye movements, perspective, and shadowiness, can impact an individual's sense of depth perception [19]. The visual cortex processes these cues according to previous experiences [16]. The subjective evaluation of these cues may increase the wide range of stereoacuity outcomes we observed in the CCVD patients in our study. Cone differentiation is controlled by competing cone pigment genes, and the mutations of these genes affect the retinal cone mosaic and the postreceptoral wiring [22-25]. Changes in either the retinal cone mosaic or the postreceptoral wiring may affect the perception of binocular disparity causing variations in the stereoacuity outcomes. However, we did not perform genotyping of the pigment genes or imaging of the cone mosaic, so we were unable to prove this suggestion.

Our study revealed that visual acuity was significantly better in patients with severe CCVD than in the control group. Consistent with our finding, Jägle et al. reported better visual acuity for multi-gene-inherited patients with CCVD than healthy individuals [22]. In the literature, it was suggested that chromatic noise, which is produced by cone photoreceptors, reduces the spatial resolution in trichromats $[22,26,27]$. Differences in the responses of $\mathrm{L}$ and $\mathrm{M}$ cones to the retinal image create a chromatic noise that negatively affects visual acuity [22,28]. According to 
the pigment replacement model, in patients with CCVD all the $\mathrm{L}$ and $\mathrm{M}$ cones are filled with the same pigment, but both the $\mathrm{L}$ and $\mathrm{M}$ cones retain their own original postreceptoral connections [24, 29]. Same spectral sensitivity of the replaced pigments and impaired $\mathrm{L}$ or $\mathrm{M}$ cone function in patients with CCVD reduces the chromatic noise, which may enhance visual acuity [22]. According to the neural loss model, both the $\mathrm{L}$ and $\mathrm{M}$ cones are filled with the same pigment and they have the same postreceptoral connections [29]. Jägle et al. and Sharpe et al. emphasized that many more postreceptoral cone pathways were allocated to luminance perception than color resulting in a better discrimination performance in patients with CCVD $[22,26]$. Differences in the developmental features of the postreceptoral cone pathways and retinal wiring may be another explanation for the superior visual acuity of dichromats.

One of the main limitations of our study is the use of the Titmus test to evaluate stereoacuity. Titmus tests use targets with fixed disparity, and patients use polarized spectacles to see the perpendicularly polarized targets [30]. The Titmus test was found to be reliable for stereoacuity of $160 \mathrm{arcsec}$ or better [8]. However, the Titmus test could overestimate stereoacuity due to monocular cues, which may cause bias in our results [30, 31]. Stereoacuity outcomes may vary depending on the type of stereo test that is used. The random dot stereo test has a modified background, which disguises the monocular cues; this advantage may influence the stereoacuity outcomes. Different transmitted light properties induced by red-green glasses used in the TNO test may also influence the stereoacuity outcomes in patients with CCVD. Further investigations with different types of stereo tests should be performed to support our results. Another limitation of our study is the use of the HRR test to detect and quantify color deficiency. Several studies noted that the HRR test is reliable, and it can be used to detect red-green color deficiencies [7, 32, 33]. However, the severity grading of the HRR test needs to be verified with the anomaloscope [7]. This might cause distortion in classifying patients according to the severity of CCVD.

In conclusion, we found that CCVD may cause decreased stereoacuity. However, the relationship between stereopsis and CCVD should be studied with further studies in a larger cohort of patients in order to reach an accurate decision.

\section{Summary}

\section{What was known before}

- Color perception were suggested to be the one of the main cues for depth perception in healthy persons.

\section{What this study adds}

- Our study showed that defective cone inputs influenced the depth perception in patients with congenital color vision deficiency.

- Our study is the first to show the stereopsis change in the absence of L-cone or M-cone photoreceptor stimulus.

\section{Compliance with ethical standards}

Conflict of interest The authors declare that they have no conflict of interest.

\section{References}

1. Cumming BG, DeAngelis GC. The physiology of stereopsis. Annu Rev Neurosci. 2001;24:203-38.

2. Martins JA, Rodrigues JM, du Buf H. Luminance, colour, viewpoint and border enhanced disparity energy model. PLoS ONE. 2015;10:e0129908.

3. Simmons DR, Kingdom FA. On the independence of chromatic and achromatic stereopsis mechanisms. Vision Res. 1997;37:1271-80.

4. Kingdom FA, Simmons DR. Stereoacuity and colour contrast. Vision Res. 1996;36:1311-9.

5. Fareed M, Anwar MA, Afzal M. Prevalence and gene frequency of color vision impairments among children of six populations from North Indian region. Genes Dis. 2015;2:211-8.

6. Deeb SS. Genetics of variation in human color vision and the retinal cone mosaic. Curr Opin Genet Dev. 2006;16:301-7.

7. Cole BL, Lian KY, Lakkis C. The new Richmond HRR pseudoisochromatic test for colour vision is better than the Ishihara test. Clin Exp Optom. 2006;89:73-80.

8. Fawcett SL, Birch EE. Validity of the Titmus and Randot circles tasks in children with known binocular vision disorders. J AAPOS. 2003;7:333-8.

9. Krauskopf J, Forte JD. Influence of chromaticity on vernier and stereo acuity. J Vis. 2002;2:6-6.

10. Jordan JR, Geisler WS, Bovik AC. Color as a source of information in the stereo correspondence process. Vision Res. 1990;30:1955-70.

11. den Ouden HE, van Ee R, de Haan EH. Colour helps to solve the binocular matching problem. J Physiol. 2005;567:665-71.

12. Cumming BG, Parker AJ. Responses of primary visual cortical neurons to binocular disparity without depth perception. Nature. 1997;389:280.

13. Watanabe M, Tanaka H, Uka T, Fujita I. Disparity-selective neurons in area V4 of macaque monkeys. J Neurophysiol. 2002;87:1960-73.

14. Ts'o DY, Roe AW, Gilbert CD. A hierarchy of the functional organization for color, form and disparity in primate visual area V2. Vision Res. 2001;41:1333-49.

15. Johnson EN, Hawken MJ, Shapley R. Cone inputs in macaque primary visual cortex. J Neurophysiol. 2004;91:2501-14.

16. Linton P. The Perception and Cognition of Visual Space. Springer; London: United Kingdom, 2017.

17. Ozolinsh M, Muizniece K. Color difference threshold of chromostereopsis induced by flat display emission. Front Psychol. 2015;6:337. 
18. Hong JY, Lee HY, Park DS, Kim CY. Depth perception enhancement based on chromostereopsis. Human Vision and Electron Imaging XVI. 2011;7865:786513.

19. Held RT, Cooper EA, Banks MS. Blur and disparity are complementary cues to depth. Curr Biol. 2012;22:426-31.

20. Flitcroft D. The interactions between chromatic aberration, defocus and stimulus chromaticity: implications for visual physiology and colorimetry. Vision Res. 1989;29:349-60.

21. McClain JE, Cacioppo A, Reising J, Koubek R. The interaction of chromostereopsis and stereopsis in stereoscopic CRT displays. Appl Ergon. 1990;21:101-6.

22. Jägle H, de Luca E, Serey L, Bach M, Sharpe LT. Visual acuity and X-linked color blindness. Graefe's Arch Clin Exp Ophthalmol. 2006;244:447.

23. Wagner-Schuman M, Neitz J, Rha J, Williams DR, Neitz M, Carroll J. Color-deficient cone mosaics associated with Xq28 opsin mutations: a stop codon versus gene deletions. Vision Res. 2010;50:2396-402.

24. Kremers J, Usui T, Scholl HP, Sharpe LT. Cone signal contributions to electroretinograms [correction of electrograms] in dichromats and trichromats. Invest Ophthalmol Vis Sci. 1999; 40:920-30.

25. Hagstrom SA, Neitz M, Neitz J. Cone pigment gene expression in individual photoreceptors and the chromatic topography of the retina. J Opt Soc Am A. 2000;17:527-37.
26. Sharpe LT, De Luca E, Hansen T, Jägle H, Gegenfurtner KR. Advantages and disadvantages of human dichromacy. J Vis. 2006;6:213-23.

27. Gordon J, Delman H, Abramov I, Tannazzo T, Scuello M. Supersensitivity in color-anomalous observers. Invest Ophtalmol Vis Sci. 2000;41:807.

28. Osorio D, Ruderman DL, Cronin TW. Estimation of errors in luminance signals encoded by primate retina resulting from sampling of natural images with red and green cones. J Opt Soc Am A. 1998;15:16-22.

29. Cicerone CM, Nerger JL. The density of cones in the fovea centralis of the human dichromat. Vision Res. 1989;29: 1587-95.

30. Fricke TR, Siderov J. Stereopsis, stereotests, and their relation to vision screening and clinical practice. Clin Exp Optom. 1997;80:165-72.

31. Ceyhan D, Mumcuoglu T, Mutlu FM, Altýnsoy HI. Comparison of TNO and titmus stereoacuity tests in healthy children. Balk Mil Med Rev. 2009;12:143-6.

32. Thiadens AA, Hoyng CB, Polling JR, Bernaerts-Biskop R, van den Born LI, Klaver CC. Accuracy of four commonly used color vision tests in the identification of cone disorders. Ophthalmic Epidemiol. 2013;20:114-21.

33. Birch J. Efficiency of the Ishihara test for identifying red-green colour deficiency. Ophthalmic Physiol Opt. 1997;17:403-8. 